\title{
Estado de la enseñanza del español como lengua extranjera en Santander ${ }^{1}$
}

\section{The state of Spanish teaching as foreign language in Santander}

\author{
Luz Mary Quintero, M.A. \\ Universidad Industrial de Santander \\ Bucaramanga, Colombia \\ Imquintero03@gmail.com \\ Leonor Avilés Arenas, M.A. \\ Universidad Industrial de Santander, \\ Bucaramanga, Colombia \\ leoavilesar@yahoo.es \\ Katherine Suárez \\ Universidad Industrial de Santander \\ Bucaramanga, Colombia \\ ikasuve@gmail.com
}

http://dx.doi.org/10.14483/udistrital.jour.calj.2014.1.a02

Received: 27-Aug-2013 / Accepted: 31-Oct-2013

To cite this article

Quintero, L. M., Avilés, L. \& Suárez, K. (2014). Estado de la enseñanza del español como lengua extranjera en Santander. Colombian Applied Linguistics Journal, 16(1). 17-28.

\section{Resumen}

Este artículo presenta los resultados parciales de un proyecto de investigación sobre el estado de la enseñanza del español lengua extranjera E/ELE en Santander en el período 2000-2011. Para el propósito de este documento nos centramos en los datos recogidos en las entrevistas y encuestas aplicadas a profesores y directivos de las instituciones participantes. Los hallazgos mostraron que hay pocas experiencias de enseñanza de ELE en Santander y las prácticas que identificamos, la mayoría se concentran en Bucaramanga y Barrancabermeja, en menor proporción. Por otra parte, encontramos que no existe evidencia de estudios de investigación formal que den cuenta del tema en Santander. Por último, se encontró que la metodología, materiales didácticos y los procesos de evaluación son siempre seleccionados o ajustados dependiendo de los propósitos de los aprendices.

Palabras Clave: Enseñanza del español, español lengua extranjera, estado del arte.

\begin{abstract}
This article presents partial results of a research project about the current state of Spanish as a foreign language in Santander between 2000 and 2011. Data were gathered by interviewing teachers and directives from the participating institutions. Besides, some documents that socialize teaching experiences were also collected. Findings show that there are few teaching experiences in Spanish as a foreign language in Santander, most of the experiences that were identified have taken place in Bucaramanga and Barrancabermeja in a lower proportion. It was also found that that there are no previous researches that give account of the field in the region. Finally, methodologies, didactic materials and evaluation and assessment processes are always selected or adapted depending on the learners' interests and needs.

Keywords: Spanish teaching, Spanish as a foreign language, state of the art.
\end{abstract}

1 Este artículo presenta los resultados parciales de un proyecto de investigación sobre el estado de la enseñanza del español lengua extranjera E/ELE en Santander en el período 2000-2011. 


\section{Introducción}

Este artículo tiene como fin dar cuenta de los resultados parciales de un trabajo investigativo realizado en el departamento de Santander, Colombia, cuyo objetivo principal fue construir un estado del arte de la enseñanza del español como lengua extranjera en Santander en el periodo 2000 - 2011. En este documento se delimitan los resultados en cuanto a las motivaciones que tiene la gente para estudiar español en la región, el perfil y rol de estudiantes y maestros, una breve caracterización de las instituciones que han ofrecido cursos de ELE, la metodología y materiales, y los procesos evaluación.

En los últimos años el español ha ocupado un lugar importante dentro de las lenguas extranjeras que las personas alrededor del mundo buscan aprender, sin embargo, según el Sistema Internacional de Certificación en Español Lengua Extrajera (SICELE), hasta el año 2010 Latinoamérica registraba un número muy bajo de estudiantes de ELE en comparación con España, país encargado de suplir el $82 \%$ de la demanda mundial de enseñanza del español. Así, los países de la región han empezado un proceso sistemático para promocionar la enseñanza/ aprendizaje de la variedad del español latinoamericano.

En el caso particular de Colombia, también ha habido un creciente interés en la enseñanza de nuestra lengua; muestra de ello es la creación de la Red Académica para el Desarrollo del Español como Lengua Extranjera EnRedELE, organización que reúne instituciones de educación formal, docentes, directivos e investigadores comprometidos con el desarrollo, la promoción y la difusión del Español como Lengua Extranjera. Entre otros propósitos, la red propone consolidar el estado del arte de ELE en los diferentes niveles de la educación formal y facilitar la conformación de alianzas entre investigadores y grupos de investigación interinstitucionales. Tales exploraciones y colectivos permitirán, por un lado, elaborar y presentar iniciativas conducentes a establecer políticas públicas y privadas en el campo del español como lengua extranjera, ELE, en el ámbito nacional e internacional y por otro, promover la cualificación de las prácticas pedagógicas y los programas de ELE. De igual manera, en cumplimiento del punto 61 del plan de gobierno de la actual presidencia de la república, se desarrolla un proyecto nacional entre las instituciones que ofrecen cursos de español para que juntas construyan un programa de ELE de alta calidad que posicione al país como un destino para el aprendizaje de la lengua. En el marco de las iniciativas antes descritas justificamos la realización de un estado del arte de ELE en el departamento de Santander.

\section{Algunas consideraciones teóricas}

El estado del arte es un estudio analítico del conocimiento acumulado en un área del saber que permite evidenciar las tendencias y los vacíos en el campo específico. Así, la construcción de un estado del arte es una potente alternativa para el desarrollo teórico, investigativo y social en la medida en que propicia la generación de nuevas comprensiones y construcciones teóricas acerca de la realidad social; motiva la creación de hipótesis comprensivas con un nivel más alto de elaboración y, por lo tanto, con mayor capacidad para orientar la investigación futura hacia la comprensión global de fenómenos sociales complejos. Igualmente, el estado del arte permite reconstruir el conocimiento acerca de la investigación social en sus aspectos tanto metodológicos como epistemológicos y teóricos; formar futuros investigadores y fundamentar líneas de investigación (Cifuentes, R. y otros, 1993). En nuestro caso, enmarcamos el proyecto del estado del arte de ELE en la línea de prácticas educativas y didáctica de lenguas extranjeras.

En cuanto a la didáctica de las lenguas extranjeras, Vez (2000) afirma que ésta recibe influencias de la psicología, la pedagogía y la lingüística moderna. En consecuencia, es un área de estudio interdisciplinar que adopta constantemente diversos aportes en búsqueda de soluciones para su desarrollo, constituyendo una trinidad teórica: ciencia pura, ciencia aplicada y práctica. Asimismo, en su naturaleza multidisciplinar, el campo de la didáctica de las lenguas extranjeras es complementado y enriquecido por los aportes de la psicolingüística, la sociolingüística, la sociología del lenguaje, las nuevas tecnologías de la comunicación (Kostina E Arboleda, 
2005), la semiótica y el análisis del discurso. La incidencia de las áreas de conocimiento citadas en la didáctica de lenguas evidencia diversos enfoques teóricos subyacentes a las prácticas de enseñanza de las lenguas extranjeras entre ellas las del español.

Una mirada general a la enseñanza de lenguas extranjeras, nos muestra una variedad de métodos y enfoques que a través del tiempo la han orientado. Sin embargo, fueron los aportes de la sociolingüística, de origen funcional, los que más han contribuido a la actual comprensión de la didáctica de las lenguas extranjeras. La sociolingüística evidencia cómo las relaciones de poder, la capacidad de interacción, el 'estatus' social y las necesidades básicas generadoras de motivación del estudiante pueden incidir en el desarrollo cognitivo y aprehensión de la lengua de un estudiante. Del mismo modo, la sociolingüística ha dado relevancia a la influencia de las culturas y las variedades lingüísticas en la enseñanza aprendizaje de una lengua extranjera en relación con el desarrollo de competencias discursivas que permitan la identificación de contextos para desarrollar actos comunicativos eficaces. Es precisamente en este marco de la sociolingüística en el que Dell Hymes propuso uno de los conceptos que más ha influido en la enseñanza de lenguas extranjeras, "la competencia comunicativa". Según el autor, las personas sabemos una serie de reglas gramaticales, pero además disponemos de una serie de habilidades discursivas y socioculturales, que nos permiten comunicarnos de un modo adecuado en cada contexto. Podemos decir con certeza que los aportes del enfoque comunicativo son los que mayor incidencia han tenido en la didáctica contemporánea de las lenguas extranjeras hasta hoy. Así, en síntesis, las actuales posturas didácticas consideran los procesos lingüísticos, cognitivos, afectivos, culturales, sociales y tecnológicos componentes cruciales en la enseñanza - aprendizaje de una lengua extranjera.

Por otra parte, discutiremos brevemente el papel del material didáctico en la enseñanza. Entendemos por material didáctico cualquier texto que presente o informe acerca de la lengua que se está aprendiendo (Tomlinson, 1998). Dicho material didáctico puede ser auténtico, adaptado o producido específicamente con fines pedagógicos. Aunque dicho concepto parece amplio, no se concibe de manera aislada sino que está limitado y vinculado con las necesidades de los usuarios, de su concepción de enseñanza-aprendizaje, con las nociones de la naturaleza de la lengua, e incluso, con el contexto cultural y la noción de evaluación.

Por tanto, como lo plantean Martín y Pueyo (2003), los materiales no se conciben de forma aislada, sino en el contexto profesional formado por creencias vigentes sobre: a) la naturaleza de la lengua, b) cómo aprenden las personas c) cómo se puede enseñar (descripción de los distintos 'métodos') y d) el entorno en el que se aprende (lo apropiado de la metodología). Son precisamente estos cuatro factores los que vamos a ver que configuran el tipo y el rol de los materiales usados para la enseñanza y, como vimos, las necesidades e intereses del aprendiz cobran una gran importancia en todos los casos reportados por los participantes en el presente trabajo.

Con respecto a la noción de evaluación es un asunto complejo que supone varios componentes que van desde la observación o reflexión de una actividad o proceso, pasan por preguntas o cuestionamientos que conllevan el análisis de la actividad o tarea a través de los datos recolectados, que nos permiten establecer diagnósticos basados en la interpretación fiable de los resultados de análisis. Lo anterior se puede realizar teniendo en cuenta diversas perspectivas de rigurosidad y de sistematicidad (Arzamendi \& Lennon, 2011).

Ahora bien, la evaluación puede ser informal o formal; en el primer caso se trata de la observación del proceso de aprendizaje en las condiciones normales de la clase e incluye la autoevaluación. Esta excluye los tests que forman parte en la evaluación formal, también se caracteriza por ser externa, aplicada a muchos alumnos en condiciones estandarizadas y cuyo fin es valorar los resultados del aprendizaje.

La comprensión del estado de la enseñanza del español como lengua extranjera en Santander permitirá la identificación de problemas, aciertos 
y la construcción de alternativas para intentar mayor efectividad en la enseñanza del idioma. Por otra parte, dará cuenta de las conformaciones teóricas, complejidad y multidisciplinariedad que compone la enseñanza de una lengua extranjera como el español.

\section{Metodología}

La presente investigación se fundamentó en el enfoque metodológico histórico - hermenéutico, desde la perspectiva de la Escuela de Frankfourt, el cual condujo a diversidad de opciones procedimentales para la comprensión de realidades, en términos de especificidad y singularidad. Esta línea de pensamiento, de naturaleza predominantemente cualitativa, admitió la interrelación e interacción entre los investigadores, los sujetos y objetos con los cuales se desarrolló la exploración. Además, no descartó la utilidad de información cuantitativa como soporte y guía para las inferencias de los investigadores (Pisso \& Euscátegui, 2003). Los métodos de investigación privilegiados fueron la etnografía y la revisión documental, por ende, como instrumentos para la obtención e interpretación de la información: libros, documentos escritos inéditos, encuestas, entrevistas semiestructuradas, registros de audio y rejillas.

Con una duración de un año y seis meses, desde el 2012, el procedimiento metodológico comprendió cuatro fases: la primera de identificación de espacios (cartografía) y fuentes de información, elaboración de criterios e instrumentos de recolección de información, consulta y registro de documentos institucionales o informales. La segunda fase de registro, compilación y clasificación de documentos, investigaciones, experiencias y material didáctico de enseñanza del español como lengua extranjera, generados en Santander y en el periodo 2000 -2011, por personas naturales, instituciones o universidades. Desde los criterios establecidos, con la población delimitada en docentes y directivos, se obtuvieron 13 entrevistas, 6 documentos (4 ponencias inéditas y dos textos con cierto valor institucional) y 6 encuestas. Cabe destacar que la indagación de las experiencias se realizó sólo con los maestros y directivos debido a que, como lo evidenció la misma investigación, gran parte de los estudiantes de ELE permanecen sólo algunos días en la región. De otra parte, el corpus citado se obtuvo sólo en el Área Metropolitana de Bucaramanga y Barrancabermeja, únicos espacios en los que se identificaron actividades de E/ELE en el lapso definido. En cuanto a las fases tercera y cuarta, se dedicaron al análisis del corpus compilado y presentación de avances y a la construcción de las conclusiones generales del estado del arte.

\section{Instrumentos}

Documentos: analizamos algunos documentos de las ponencias presentadas por docentes en el marco de los encuentros regionales del sistema Internacional de Certificación del Español como Lengua extranjera, SICELE. Es pertinente aclarar que según los informantes entre el período 2000-2011 no se registraron proyectos de investigación que dieran cuenta de las experiencias tanto de enseñanza como de aprendizaje en el tema en la región. En cuanto a los materiales, programas, políticas y demás documentos no fue posible acceder a gran parte de ellos, creemos que dado el carácter inédito de los mismos, las instituciones se abstienen de compartirlos.

Encuestas: las encuestas tuvieron como propósito recoger información demográfica sobre los aprendices de ELE que han venido a las instituciones. La información obtenida pretendió complementar la información sobre el perfil de estudiante de ELE en la región. La encuesta fue diligenciada solo por los directivos.

Entrevistas: La entrevista se convirtió en el instrumento más importante de recolección de datos. Se realizaron entrevistas semi-estructuradas a directivos y docentes de las instituciones participantes.

\section{Contexto y Participantes}

El presente estudio se realizó con cinco instituciones de educación de las cuales tres son universidades, una pública y dos privadas, y dos institutos de educación no formal. En cuanto a las personas que nos proporcionaron los datos, tenemos cuatro directivos y nueve docentes. Seis docentes hacen parte del equipo docente de las instituciones anteriormente descritas; los otros tres docentes son instructores privados, uno de 
ellos ofrece cursos privados personalizados en Barrancabermeja. En el periodo delimitado, fuera del Área Metropolitana de Bucaramanga sólo se registraron experiencias en Barrancabermeja. Las instituciones y personas se escogieron bajo dos criterios: aquellas que voluntariamente manifestaron su voluntad de hacer parte del proyecto, y que a su vez hubieran ofrecido cursos de ELE en Santander en el período objeto de estudio (2000-2011).

También cabe destacar que existe un instituto localizado en el Socorro-Santander que ofrece cursos de ELE, el cual ha tenido una buena acogida. No obstante, no se incluyó dentro del estudio porque fue creado después del lapso objeto de estudio de este proyecto.

\section{Hallazgos}

A continuación presentamos el análisis de los datos y los mayores hallazgos organizados en las siguientes categorías planteadas a manera de pregunta: ¿por qué se viene a aprender español en Santander?, ¿quiénes vienen a aprender?, cंquiénes enseñan?, ċuáles métodos y materiales didácticos se han utilizado?, ccómo se ha evaluado el proceso de aprendizaje? Hemos usado algunos códigos E1, E2, E3 etc, que representan los informantes a fin de conservar su anonimato.

\section{¿Por qué se viene a estudiar ELE en Santander?}

En el estudio encontramos que las razones por las cuales los extranjeros deciden venir a aprender español a Santander son variadas. Sin embargo, las podemos agrupar en cuatro: el atractivo turístico y cultural de la región, intercambios académicos con universidades, intereses laborales y aunque parezca menos llamativo, encontramos que unas cuantas personas vienen por motivaciones sentimentales. A continuación expondremos en detalle cada una.

Santander resulta ser una zona con un alto atractivo turístico, especialmente en lo que se refiere a actividades y planes desarrollados en ambientes ecológicos y naturales y a los deportes extremos. El mayor atractivo no es precisamente la ciudad capitalBucaramanga, sino los municipios cercanos. Los resultados concuerdan con lo señalado por Agray (2007) quien sostiene que fue la industria del turismo la que impulsó el desarrollo del ELE en el país en sus inicios y se consolidó, de manera especial en la Costa Atlántica y en Bogotá.

"[les llama la atención] la cultura latina, les gusta mucho el color, el hecho de ser diferentes, la alegría, todo esto les llama la atención, a ellos lo que les interesa es visitar, como somos tan diferentes y estamos tan lejos con respecto a Australia, les interesa aprender más de nuestra cultura." (E3).

"ellos [los aprendices] quieren conocer la gente, conocer la cultura, familiarizarse con el idioma, practicarlo. Pues es una zona muy cultural, la naturaleza, de pronto salir de grandes ciudades, venir a áreas como el Parque Chicamocha o los pueblos como San Gil, pueden tener actividades de torrentismo, rafting, y ellos pueden estar como más en contacto con la naturaleza." (E6).

Resulta interesante identificar que el turismo, en especial de naturaleza y de aventura, sea una de las principales razones por la que los extranjeros escogen a Santander para aprender el idioma. Al parecer a ellos les resulta atractivo combinar el aprendizaje del idioma con la exploración de los lugares y atractivos culturales de la región. Desde esta perspectiva, es preciso proyectar y ofertar proyectos de aprendizaje de la lengua en los que se involucre el atractivo turístico de Santander, puesto este es uno de los proyectos estratégicos para la región y el país, según lo expresado por la Viceministra de Turismo quien señala: "recientemente con el programa de transformación productiva hemos desarrollado un plan de negocios para mejorar las condiciones del sector [En Santander] y buscar potencializar a nuestro país en este tipo de turismo que le vemos un gran potencial" (Acuña (2013, mayo, 12).

La segunda razón para tomar clases de español en la región se relaciona con los intercambios académicou, tanto de estudiantes como profesores provenientes de distintos países. Vemos que de 
manera creciente y progresiva las universidades en su interés por internacionalizar sus programas, han creado estrategias para motivar la visita de estudiantes y docentes con fines académicos. Así lo confirman algunos entrevistados:

"Ellos vienen por pasantías cortas. vienen docentes, vienen estudiantes en intercambio semestral, académico. La mayoría estudia administración de empresas, administración turística y hotelera. Hay unas niñas de enfermería, pues tenemos un convenio con una universidad... han venido también personas a enfermería." (E5)

"ellos vienen de intercambio y más que todo son de Francia y de Alemania. Tener una materia de español, les permite a ellos como profundizar o simplemente ser más fluidos en las clases de español cuando ellos tiene que ir a las clases magistrales." (E10)

Vemos de manera muy positiva que la movilidad de las universidades de la región no ocurre solamente por parte de los estudiantes y docentes de la región que viajan hacia otros países, sino que paulatinamente instituciones de todos los continentes vienen con intereses académicos a las universidades de la región. Este fenómeno a su vez representa una necesidad por aprender o mejorar la competencia en el idioma local, lo que representa una buena oportunidad para la enseñanza del español. Cabe resaltar que en la mayoría de los casos los aprendices en intercambios académicos llegan con un conocimiento intermedio del español que les permite comunicarse y comprender a otros interlocutores en el plano académico; sin embargo, según una de las personas entrevistadas, no se sienten lo suficientemente seguros al momento de escribir textos académicos o de interactuar oralmente, razón por la que deciden tomar cursos para afianzar su conocimiento.

En cuanto a la tercera motivación, asuntos laborales, debemos resaltar que la mayoría está relacionada con la explotación petrolífera o con centros de investigación sobre temáticas similares. Indudablemente, Santander tiene un atractivo laboral y de negocios gracias a la explotación minera de hidrocarburos y oro en varias regiones de la zona. Es notorio que las necesidades e intereses de este tipo de aprendices son muy puntuales y contextualizados, según lo manifestado por algunos entrevistados:

“...ahí un mes o más o menos porque la estadía de ellos [los americanos] es muy corta y lo que hay que enseñarles es un español práctico de lo que van a usar en una planta de producción o en un campo petrolero." (E13)

Yo creo que la gente que decide estudiar español lo hace por fines muy concretos, normalmente están ligados al desarrollo laboral." (E4)

Finalmente, la última razón que expondremos tiene que ver con los vínculos sentimentales o afectivos de algunos de los aprendices. Algunos de ellos vienen a vivir en Santander y la gran mayoría aprovecha las visitas cortas para aprender o mejorar el idioma.

“...otras ya en un nivel más afectivo (estaban casados o con proyectos de casarse con colombianos), algunas personas quieren casarse con alguna latina." ( E3)

\section{¿Quiénes vienen a aprender?}

En cuanto a los lugares de origen de los aprendices, podemos decir que ha habido un flujo significativo de los cinco continentes. De mayor a menor, las siguientes son las nacionalidades que resultaron de los datos recogidos en las encuestas y las entrevistas: Primero, Francia, Canadá, Estados Unidos y Alemania; seguidos por China, Japón, Corea Australia, Suecia, Brasil, Lituania, India y Noruega. La edad predominante de los aprendices es 26 a 30 años; seguido por 31 a 35 y 21 a 25 años. En cuanto al sexo, se puede decir que se ha distribuido en forma equitativa entre hombres y mujeres, aunque en algunas instituciones se resalta más la presencia de los hombres, en especial en el campo de la minería y explotación de hidrocarburos.

Ahora nos referiremos a la actuación académica de los aprendices. Con base en los datos recogidos en la encuesta, la mayoría de los profesores y 
directivos coinciden en que los aprendices de ELE son disciplinados, constantes, reflexivos y sociables. Sólo un participante señaló que los participantes fueron poco sociables. Lo anterior se puede ilustrar en palabras de una participante:

"Lo que puedo decir es que son personas muy autónomas... muy disciplinadas que aprovechan muchísimo el tiempo... no esperan un estímulo para hacer las cosas, ni tampoco esperan un estímulo como de reconocimiento a lo que han logrado. Tienen las metas muy claras y simplemente hacen, son personas disciplinadas, pero no sólo en el sentido que hagan las cosas por su cuenta y su voluntad sino que hacen lo que se les pide hacer. Ellos buscan por su cuenta, ellos piden un poco más." (E11).

"El europeo es un extraordinario aprendiz de lengua extraordinario, nosotros vemos que ellos logran el desarrollo a una velocidad increíble." (E6)

De igual manera, los aprendices se caracterizan por tener un conocimiento previo de la lengua española, afirmación común en casi todos los entrevistados. Esto a su vez hace que los materiales, las actividades y la evaluación tengan que ser ajustados acorde a las necesidades de cada grupo, como bien lo veremos más adelante. Este perfil de estudiante autónomo, responsable y con alta motivación, según los docentes entrevistados, hace que se vean resultados rápidos. Ahora veamos cuál es el perfil de los docentes que han enseñado ELE en Santander.

\section{¿Quiénes enseñan ELE en Santander?}

El campo de formación de los docentes con mayor predominancia es el área de lenguas, de manera que encontramos: licenciados en idiomas, licenciados en español y literatura y en menor cantidad, en otras áreas del conocimiento como finanzas y administración. Sólo un profesor posee un título de maestría en la enseñanza de ELE y algunos han tomado un curso corto o un diplomado en el tema. Sin embargo, todos los docentes son competentes en al menos una lengua extranjera, en su mayoría en inglés. Según los docentes y directivos, conocer al menos una lengua extranjera representa una ventaja al momento de enseñar el español pues le permite al profesor usar el inglés o incluso el francés para explicar lo que el aprendiz no logra comprender en español. Por otro lado, casi la totalidad de los docentes tienen experiencia en la enseñanza de lenguas extranjeras, aún aquellos cuya profesión no está relacionada con la enseñanza de lenguas. En varios casos los docentes han vivido en otros países.

En cuanto a la actuación o papel que desempeña el docente en el aula de clase cabe destacar que la mayoría se considera un guía, un orientador, dado que es común encontrar que los aprendices tienen un buen conocimiento del español. También se subrayó el rol del docente como un mediador de la cultura local y la lengua. Este último aspecto revela que el profesor ha asumido un rol que trasciende su preocupación por el uso gramatical "correcto" del idioma, para convertirse en alguien que ayuda a comprender el uso de éste en un determinado contexto sociocultural y cómo éste influye y genera unas variedades lingüísticas, que de ninguna manera "distorsionan" el idioma, sino que representan la riqueza y los aportes de las culturas. Cuando se preguntó por el papel que desempeña como docente de ELE una participante afirmó:

"Bueno yo creo que mi papel ha sido más de guía y de promotora del manejo correcto de la lengua pero en ciertas habilidades. No el papel como de iniciar un proceso, porque las personas con las que he trabajado ya tenían un proceso desarrollado. De pronto ellos vinieron más con el propósito de seguir desarrollando ciertas habilidades." (E11)

"Pues el papel más allá de enseñar un idioma es transmitir una cultura, eso es lo que casi siempre hago. Saco mucho tiempo de mis clases para hablar bien bastante de lo que es la cultura latinoamericana $\mathrm{y}$ de transmitir sobre todo que no hay sociedades ni culturas superiores sino que hay sociedades y culturas diferentes, transmitir ese concepto de no es malo, no es feo sino es diferente." (E1) 
La postura de algunos docentes es similar a la expuesta por Hernández y Maite (2004) quienes afirman que la competencia comunicativa en una lengua va más allá de saber responder a una serie de funciones comunicativas aisladas. Al explicar la lengua como construcción social e individual, sus funciones y funcionamiento se propone considerar la cultura, interacción y competencias que tiene el aprendiz tanto de su lengua materna como de la lengua foránea las cuales configuran la razón de su aprendizaje.

Por otra parte, la mayoría de los entrevistados manifiesta no estar familiarizados con las políticas locales, nacionales o internacionales relacionadas con la enseñanza de español lengua extranjera. Solo dos participantes expresan conocer algunas por distintas razones. Uno señala que:

"una política que se tiene es el Marco Común Europeo, la Casa Cervantes para cómo e enseñar los parámetros de español, sí, uno se guía por esos marcos hasta ahora porque no hemos hecho un marco común latino, donde nosotros podamos tener nuestras propias funciones, nuestras propias leyes." (E10)

Ninguno de los entrevistados explica de qué manera cree que las políticas inciden en el ejercicio de la enseñanza, hechos que llaman la atención si tenemos en cuenta que en el país se han socializado políticas en relación con el SICELE y algunas iniciativas para la promoción del aprendizaje. Sin embargo, al parecer tales iniciativas no han tenido impacto alguno en el plano de la práctica docente.

\section{¿Qué instituciones han ofrecido ELE?}

Después de una búsqueda cuidadosa, encontramos que en la región no había mayor número de instituciones y personas dedicadas a la enseñanza del español como lengua extranjera. Así, identificamos en Bucaramanga tres universidades, una pública y dos privadas, que ofrecen cursos a través de sus institutos de lenguas. En una universidad sólo se ofrecen cursos en la modalidad de intercambio académico durante un año lo cual también les da la oportunidad de hacer un intercambio cultural; existe un instituto que ofrece solo cursos de español a extranjeros; y, una universidad que ofrece cursos de idiomas a través de su instituto de lenguas, entre ellos el español. En Barrancabermeja encontramos que únicamente se brinda servicio de clases particulares.

\section{¿Cómo y con qué materiales se ha enseñado ELE?}

A continuación daremos una mirada global a la metodología y a los materiales que se han usado para la enseñanza de ELE en Santander.

Es preciso resaltar que si bien es cierto algunos profesores no usan la terminología técnica para referirse a los métodos o a los enfoques, sí describen en detalle la forma como se enseña, lo cual nos permitió inferir de acuerdo a los planteamientos teóricos que la mayoría de los entrevistados prefieren los enfoques procesuales o comunicativos. En la Entrevista se enuncia en los siguientes apartados:

"Utilizo el método comunicativo, porque está más acorde con el uso del idioma en contextos reales de comunicación: cómo comprar una comida, cómo contestar el teléfono, etc." (E12)

"El método ha sido un enfoque como comunicativo, [...] ellos por lo menos van a vacaciones y ellos cuando llegan, con base en el tema que hemos visto, me cuenten primero en forma escrita, después le cuenten a los muchachos en forma oral lo que han hecho" (E10)

En la misma línea del enfoque comunicativo y procesual hallamos que algunos participantes explicitan el uso del enfoque por tareas o el aprendizaje orientado a la acción:

"Hablábamos más de un método como accional, en donde el hacer, la acción como tal era más importante que estar encerrados en el aula de clase $y$ en ese sentido es que enfocamos todo y por eso fue que llevamos al estudiante a lugares donde él sintiera conflictos en relación con aprender la lengua."(E1) 
"La vinculación de la enseñanza a la inmersión del estudiante en la cultura local, es muy común. Se trabajaba con el estudiante llevándola al mercado local, al mercado de las pulgas, a los eventos públicos." (E8)

La visión que presentan las anteriores afirmaciones están relacionadas con el bien conocido enfoque comunicativo, que hace un gran énfasis en la enseñanza de la lengua en un contexto que promueve la comunicación y la creación auténticas de sentido (Richards \& Rodgers, 2001). Es decir, no es suficiente con que el aprendiz sea capaz de analizar el sistema linguístico, es indispensable que sea capaz de comunicarse en una variedad de actos de habla. Por lo tanto, la lengua se concibe como discurso que se materializa y desarrolla en las prácticas sociales y culturales de los sujetos hablantes.

Como antes anotamos, los demás entrevistados no hacen explícita la metodología aplicada, es decir no mencionan un nombre específico, sin embargo, es posible identificar el uso de dichos métodos por los conceptos mencionados tales como "competencias", "actividades prácticas", etc. Podemos afirmar que un número significativo de entrevistados hizo un fuerte énfasis en la enseñanza comunicativa. Del mismo modo, los docentes manifestaron haber incluido las cuatro habilidades de la lengua, con un mayor énfasis en una o algunas habilidades dependiendo siempre del propósito e interés del estudiante.

A la par se observa que algunos docentes adoptan una postura ecléctica en cuanto a la metodología, pues se evidencia la mezcla entre el enfoque comunicativo con los principios de otros métodos como el audio-lingual o simplemente se mezclan técnicas de métodos distintos, que se explicita en el siguiente extracto:

"trato de utilizar mucho el método comunicativo y aspectos del método audio-lingual y otros contenidos, pero como tal, no puedo decir que enseñé o he utilizado cien por ciento este método." (E1)

Podemos decir que la misma diversidad de propósitos, necesidades y aún estilos de los aprendices, hacen que el docente adopte múltiples metodologías de enseñanza que ayuden a todos a alcanzar sus objetivos particulares. Esto no es una tarea fácil para un docente pues demanda conocimiento profundo de las distintas formas de enseñar una lengua extranjera así como también diferentes desempeños en el desarrollo de un curso o incluso de una lección.

Al igual que la metodología seleccionada, los materiales didácticos fueron creados o adaptados de acuerdo con las necesidades de los aprendices y el entorno cultural. En ningún caso se usó un libro de texto exclusivo para orientar la instrucción. Encontramos que la producción de materiales didácticos depende de los propósitos de los aprendices identificados en una primera exploración de necesidades de aprendizaje, y dado que estas son tan diversas los profesores debieron crear sus propios materiales como guías, cuadernillos o fichas. La creación de los materiales puede representar un trabajo arduo desde el punto de vista del profesor, pero es una gran ventaja para el estudiante pues en general los materiales publicados no conectan ni hacen uso completo del idioma fuera del aula, mientras que el profesor conoce el contexto de uso y lo incorpora en sus materiales. Así lo indicaron los profesores:

“...materiales que sacaba, que escribía yo mismo, escribía cosas así de historia, creaba actividades gramaticales..." (E1)

"Uso material de producción propia; por ejemplo, busco un recorte de un periódico, hago preguntas. También el texto. Si es una salida, elaboro fichas al respecto..." (E12)

De la misma manera, no sólo se elabora el material, sino que en muchos casos se adapta de diferentes fuentes, dependiendo, como ya se dijo, de los propósitos del aprendiz o del entorno en el que se está. Es por esto que encontramos una gran variedad de materiales que ayudan a mediar el proceso de enseñanza-aprendizaje tal como se precisa en las entrevistas: 
"El tipo de material básicamente depende de las necesidades de usuario, generalmente ellos quieren un español práctico, concreto, hablado..." (E3)

"El material mencionado anteriormente (es tomado) del contexto de la ciudad: almacenes, personas, transporte, objetos reales, revistas, televisión, películas, libros, cuentos, y el libro texto, etc". (E12)

De manera semejante, se enuncia la adaptación de materiales siguiendo pautas de materiales elaborados para la enseñanza de otros idiomas, con preferencia por el inglés y el francés. En este caso, no se adapta el contenido de un material sino que se toma la estructura del mismo, el tipo ejercicios secuencias, etc. para diseñar el material propio:

:...básicamente es copiar de los materiales de inglés y adaptarlos al español... lo que hacía era mirar los libros de enseñanza de inglés y copiar el mismo proceso, ese era el material didáctico, utilizar el libro de inglés y de ahí copiarlos los mismos procesos, a los mismos ejercicios, acomodar los ejercicios y las actividades a la enseñanza del español... también de francés como modelo para generar materiales didácticos es una opción y esa la utilizaba bastante." (E1)

Estos hallazgos, que coinciden con lo encontrado por De Mejía, López, Cruz, Flaborea y Guzmán (2009), revelan que en muchos casos los mismos profesores de ELE son los encargados de diseñar y obtener todo lo que necesitan para las actividades de clase. Por lo tanto, los autores enfatizan en la necesidad de elaborar más materiales didácticos por parte de las instituciones que estén acordes con la realidad colombiana y con la realidad de los alumnos, así como materiales que involucren nuevas tecnologías, como páginas web, recursos audiovisuales, entre otros. En la misma línea García, D., García, J. y Buitrago Y. (2011) plantean que la falta de divulgación hace que muchas veces las mismas instituciones desconozcan lo que se está generando localmente y tiendan a tomar como referencia aquellos materiales creados en el exterior, especialmente en España. Este hecho nos lleva a pensar que la inexistencia de materiales didácticos propios no permite el reconocimiento del español latinoamericano.

\section{¿Cómo se evaluó el proceso de aprendizaje?}

Un número significativo de las experiencias reportadas en este estudio utilizaron diversas formas de evaluación de acuerdo con los propósitos de los estudiantes. Fue común hallar que en la mayor parte de los aprendices de ELE no vienen con la intención de certificarse en la lengua, por consiguiente el propósito, tipo, frecuencia y contenido de la evaluación también están sujetos a adaptaciones pues ninguno de ellos requiere estandarización. Por otro lado, se puede decir que los profesores tienen un alto nivel de autonomía para decidir sobre cómo, qué y cuándo evaluar. Este último rasgo está en consonancia con la hallado por (De Mejía et al., 2009) quienes indican que "durante el curso hay mucha flexibilidad para evaluar, el criterio del profesor tiene parte importante en este sentido" (p. 42).

El tipo de evaluación y su frecuencia variaron de acuerdo con cada contexto de enseñanza y con los propósitos del aprendiz. Entre los tipos de evaluación más frecuentes encontramos: evaluación de diagnóstico, la cual se hace al comienzo del curso para establecer el nivel actual de competencia del aprendiz y generalmente a partir de ella se plantea la enseñanza (De Mejía et al., 2009). De otra parte, los autores explican el concepto de evaluación directa e indirecta: la evaluación directa es la evaluación de lo que está haciendo realmente el alumno que se somete a examen. La evaluación indirecta por su parte, utiliza una prueba, habitualmente en papel, que a menudo evalúa las destrezas.

Sin embargo, es vital destacar que la mayor parte de las evaluaciones tendieron a ser informales y a concentrarse en el análisis de la competencia y el desempeño globales del aprendiz más no en una nota cuantitativa.

"Yo creo que evaluaba diario, yo a diario iba mirando cómo iban progresando en que fallaban, lo que yo veía que no habían comprendido volvía otra vez sobre eso de otra manera, de otra perspectiva, pero siempre buscando que fuera interesante para ellos, que ellos vieran la necesidad de comunicarse bien." (E8) 
Sólo en tres casos se mencionaron exámenes y quices con porcentajes para cada uno de ellos. Estos rasgos de la evaluación se correlacionan con los reportados por (De Mejía et al., 2009) en los que se resaltan la flexibilidad que tiene el profesor para decidir a juicio propio sobre la frecuencia, los temas y el tipo de evaluación. Así, identificamos una rica amalgama de tipos y frecuencia de evaluación que van desde interacciones reales con personas de las comunidades, hasta presentaciones un poco más formales en el aula sobre temáticas diversas, escritura de textos pasando por exámenes formales, aunque menos comunes. En cuanto a la frecuencia de la evaluación, se puede decir que algunos profesores que evaluaban día a día, otros solo al final del curso y otros que evaluaban al estudiante en la capacidad de desenvolverse en el contexto de uso del idioma, es decir en la interacción con los hablantes. El siguiente extracto ilustra las ideas arriba expuestas:

"En cuanto a la evaluación arriba mencionada...probablemente una o dos veces por semana...no puedo decir cada semana pero si al menos una vez por semana dos veces por semana buscaba esa opción." (E1)

Destacamos que con frecuencia la evaluación se realiza para dar cuenta del aprendizaje de los estudiantes más no para evaluar el proceso de enseñanza o los materiales didácticos, entre otros. Es notorio que persiste la visión de la evaluación sólo desde la perspectiva del aprendizaje o los logros del estudiante más no una evaluación de las metodologías, los materiales y otros elementos que merecen ser revisados dentro de la enseñanza y el aprendizaje.

\section{Conclusiones}

A manera de conclusión queremos comenzar por manifestar que contrario a nuestras expectativas iniciales como investigadores, hallamos que hay pocas experiencias de enseñanza de ELE en Santander. Todas las iniciativas a excepción de Barrancabermeja y El Socorro, se concentran en Bucaramanga. Este hecho llama la atención teniendo en cuenta que los extranjeros prefieren visitar e incluso residir en pueblos más pequeños y con atractivos turísticos. En lo que se refiere a materiales didácticos y demás documentos escritos no fue posible tener acceso porque no existen o quizás, dado al carácter inédito de los mismos, las personas y las instituciones prefieren mantenerlos en reserva. Esto a su vez hace que las experiencias no sean visibles en la región

Otra gran reflexión final está relacionada con el papel central que ocupa el aprendiz en la totalidad de las experiencias relacionadas en el proyecto. Todos los cursos, sin excepción, son planeados a partir de las necesidades e intereses del aprendiz, de ahí que todas las demás decisiones curriculares que se tomen en cuanto a objetivos, metodologías, materiales y evaluación varíen siempre con el objeto de satisfacer las necesidades del aprendiz. Es decir, que podemos sin temor a dudas afirmar que el currículo está centrado en el estudiante. Postura que coincide con lo expuesto por Richards (2001) quien considera que el primer paso en la construcción de un currículo debe partir del análisis de las necesidades reales del aprendiz.

En cuanto a las motivaciones para aprender ELE en Santander encontramos que hay variadas, pero predomina el tema del turismo, seguido por los intercambios académicos asuntos de naturaleza sentimental. Vale pena pensar en proyectos más ambiciosos que promuevan el aprendizaje del idioma y el turismo y para que esto sea posible habría que pensar en alianzas entre los sectores académico y turístico.

Respecto a cómo se enseñó podemos concluir que se privilegian los principios del enfoque comunicativo que hacen énfasis en los procesos; también varios de los procedimientos metodológicos de enseñanza presentados tienden a ser eclécticos, pues el maestro debe adaptar su metodología dependiendo del aprendiz y de las necesidades e intereses del mismo.

En consonancia con el componente metodológico encontramos que los materiales didácticos usados por los maestros promueven el desarrollo de la competencia comunicativa a través de tareas significativas. Y, al igual que las metodologías, los materiales propenden por el desarrollo de las cuatro habilidades de la lengua, sin embargo alguna o algunas de ellas resultan privilegiadas dependiendo de las necesidades de los aprendices.

En el mismo sentido, la evaluación tiene una gran variedad de tipos, frecuencias e instrumentos. El 
profesor cuenta con un alto grado de autonomía para decidir sobre la evaluación. En general, encontramos un interés por la evaluación formativa que resalta la atención en el proceso más que en una nota final o el resultado mismo.Finalmente, resaltamos que es de vital importancia continuar con iniciativas de investigación que difundan los avances en el campo de la enseñanza e investigación de ELE en el país así como la creación de materiales didácticos propios.

\section{Referencias}

Acuña, N. (2013, mayo, 12). En Santander se invertirá en el turismo de aventura y natural. Vanguardia. com. Recuperado de http://www.vanguardia.com/ santander/guanenta/207877-en-santander-seinvertira-en-el-turismo-de-aventura-y-natural).

Agray, N. (2007). El español como lengua extranjera, perspectivas, retos y oportunidades. Una visión panorámica. Memorias I Encuentro Regional de ELE.

Arzamendi, J. E Lennon, A., (2011). Evaluación del proceso de aprendizaje de lengua. Barcelona, Funiber.

Cifuentes, M. R. Osorio, F. E Morales, M. (1993) Una Perspectiva Hermenéutica para la construcción de estados del arte. Manizales, Universidad de Caldas.

García, D., García, J. \& Buitrago Y. (2011). Estado del Arte de ELE en Colombia, Una Mirada Material Didáctico, Variedades. Marco ELE Revista de didáctica. ELE, 13.

Hernández, M. E Villalba, F. (2004). Diseño curricular para la enseñanza del español L2 en contextos escolares. En Fortele. Formación telemática en la enseñanza del español como lengua extranjera. Consejería de Educación y Cultura. Dirección general de enseñanzas escolares. 1-11.
Kostina, I. y Arboleda, A. (2005). La Didáctica de Lenguas Extranjeras: un campo interdisciplinar, multidisciplinar y multidimensional. En Vásquez, F. (ed.) (2005) La Didáctica de la Lengua Extranjera. Estado de la discusión en Colombia. Cali, Unidad de Artes Gráficas.

Martín, I. y Pueyo, S. (2003). Formación de Profesores de Español como Lengua Extranjera, asignatura: Creación, Adaptación y Evaluación de Materiales y Recursos. Funiber.

De Mejía, A. M., López, A.A., Cruz, F., Flaborea, R., \& Guzmán, M. (2009). Informe del estudio sobre los exámenes en español como lengua extranjera administrados en Colombia. Centro de Investigación y Formación en Educación, (CIFE) Instituto Caro y Cuervo. Bogotá: Universidad de los Andes.

Pisso, F. y Euscátegui R. (2003). Investigación educativa. Popayán: Universidad del Cauca.

Red Académica para el Desarrollo del Español como Lenredele.org.co/archives/93.

Richards, J. E Rodgers., T. S. (2001). Enfoques y métodos en la enseñanza de idiomas. Cambridge: Cambridge University Press.

Richards, (2001). Curriculum development in language teaching. Cambridge: Cambridge University press.

Tomlinson, B. (ed.) (1998): New Directions in L2 Materials Development. Cambridge: Cambridge University Press.

Vez, J. M., (2000). Fundamentos lingüísticos en la enseñanza de lenguas extranjeras. Barcelona, Ariel Lenguas Modernas.

\section{THE AUTHORS}

LUZ MARY QUINTERO, M.A. in Applied Linguistics, specialist in University Teaching and B.A. in Languages. She teaches in Escuela de Idiomas at Universidad Industrial de Santander.

LEONOR AVILÉS ARENAS, M.A. in Languages, M.A. in Semiological Studies at Universidad Industrial de Santander and M.A in Formación para la Enseñanza del Español como Lengua Extranjera at Universidad de León, Spain.

KATHERINE SUÁREZ, B.A. student in Spanish and Literature at Universidad Industrial de Santander 